\title{
Management of Small Cell Neuroendocrine Carcinoma of Urinary Bladder, A Case Series from Northern Pakistan
}

\author{
Humera Mahmood ${ }^{1}$, Hadia Fatima ${ }^{2}$, Mohammad Faheem ${ }^{3}$ \\ ${ }^{1}$ Head of department Oncology, Atomic Energy Cancer Hospital, Pakistan \\ ${ }^{2}$ Resident Oncology, Atomic Energy Cancer Hospital, Pakistan \\ ${ }^{3}$ Chief Oncologist and Director, Atomic Energy Cancer Hospital, Pakistan
}

Submission: December 19, 2017; Published: January 08, 2018

"Correspondence Address: Hadia Fatima, Resident Oncology, Atomic Energy Cancer Hospital, Nuclear Medicine Oncology and Radiotherapy Institute (NORI), Islamabad, Pakistan, Tel: +92 334 5263220; Fax no: +92-51-9260616; Email: hadia.fatima@hotmail.com

\begin{abstract}
Small cell neuroendocrine carcinoma is a rare histological variant of urinary bladder malignancies. The clinical behavior is aggressive and prognostic outcome is very poor in such cases. Patient's presentation may correspond to bladder tumors but may exhibit characteristic features of neuroendocrine malignancies. The tumor is diagnosed histologically and through immunohistochemical stains. This tumor is usually picked up in advanced stages. Cisplatin and etoposide is the first line chemotherapy usually administered in these cases. Patients not responding to chemotherapy might benefit from palliative radiotherapy. Three of these cases have been reported in this case series emphasizing the role of correct diagnosis of Small cell bladder tumors. All of these patients were diagnosed initially as urothelial tumors. They were picked up later in advanced stages. Patients were managed at the oncology department initially with combination chemotherapy but most of them had a relapse and had to be planned for locoregional palliative radiotherapy
\end{abstract}

Keywords: Bladder cancer; Cisplatin; Etoposide; Neuroendocrine tumor; Small cell carcinoma

\section{Introduction}

Among urinary bladder malignancies, neuroendocrine tumors comprise of a very small percentage1. They range from low grade carcinoid tumors to high grade small and large cell neuroendocrine carcinomas. Small cell neuroendocrine carcinomas (SCNC) comprise of less than $1 \%$ of all the primary bladder malignancies [1]. These tumors are mostly reported in elderly population with male predominance [2]. Pure forms of SCNC are rare and less likely associated with paraneoplastic syndromes [2]. Patients usually present in stage III and IV with complaint of painless hematuria [3]. They are aggressive tumors with poor prognostic outcome [3]. Median survival in such patients is up to 23 months [3]. Up to 400 cases have been reported in the literature so far with 5 years overall survival of $8 \%$ for both pure and mixed forms [2]. Few such cases have previously been reported in Pakistan but in all these cases only surgical management has been discussed but the role of chemotherapy and radiotherapy has not been addressed. We hereby report a case series of three middle aged male patients of small cell neuroendocrine tumor who presented at Oncology department of Atomic Energy Cancer Hospital, Nuclear Medicine Oncology and Radiotherapy Institute (AECH, NORI) and received multimodality treatment with surgery, chemotherapy and radiotherapy.

\section{Case 1}

A 41 year old male, addicted to betel nut with past medical history of pulmonary tuberculosis and past surgical history of bladder stones removal and no family history of malignancy, presented with the complaint of dysuria for one year. Ultrasound pelvis showed a mass in right lateral wall of urinary bladder of 24 x $18 \mathrm{~mm}$. Cystoscopy and TURBT was done and histopathology revealed invasive papillary urothelial carcinoma. Lamina propria was involved but the muscle was spared. Patient was advised three monthly cystoscopy and regular follow up by the urologist but he didn't turn up.

He remained well for six months but became symptomatic again and presented at Oncology department, AECH NORI with 
the complaints of dysuria and passage of blood clots in urine. Patient had good performance status (ECOG 1) and abdominal examination was unremarkable except for well healed suprapubic surgical scar. CT urogram was done that showed an ill-defined, enhancing lesion measuring 9.6 × 9.3 x $6.5 \mathrm{~cm}$ (CC x T $\mathrm{x}$ AP) arising from left lateral and anterior wall of urinary bladder (Figure 1A). The growth had indistinct interface with prostate and left lateral pelvic wall. Multiple lymph nodes were noted, the largest $1.9 \mathrm{~cm}$ along the internal iliac vessel. A suspicious sclerotic lesion was noted at DV12. Bone scan correlation showed no scintigraphic evidence of osseous metastasis. The disease was staged as T4bN1M0. Repeat cystoscopy and TURBT was done. Histopathology report showed high grade urothelial carcinoma with muscle invasion.
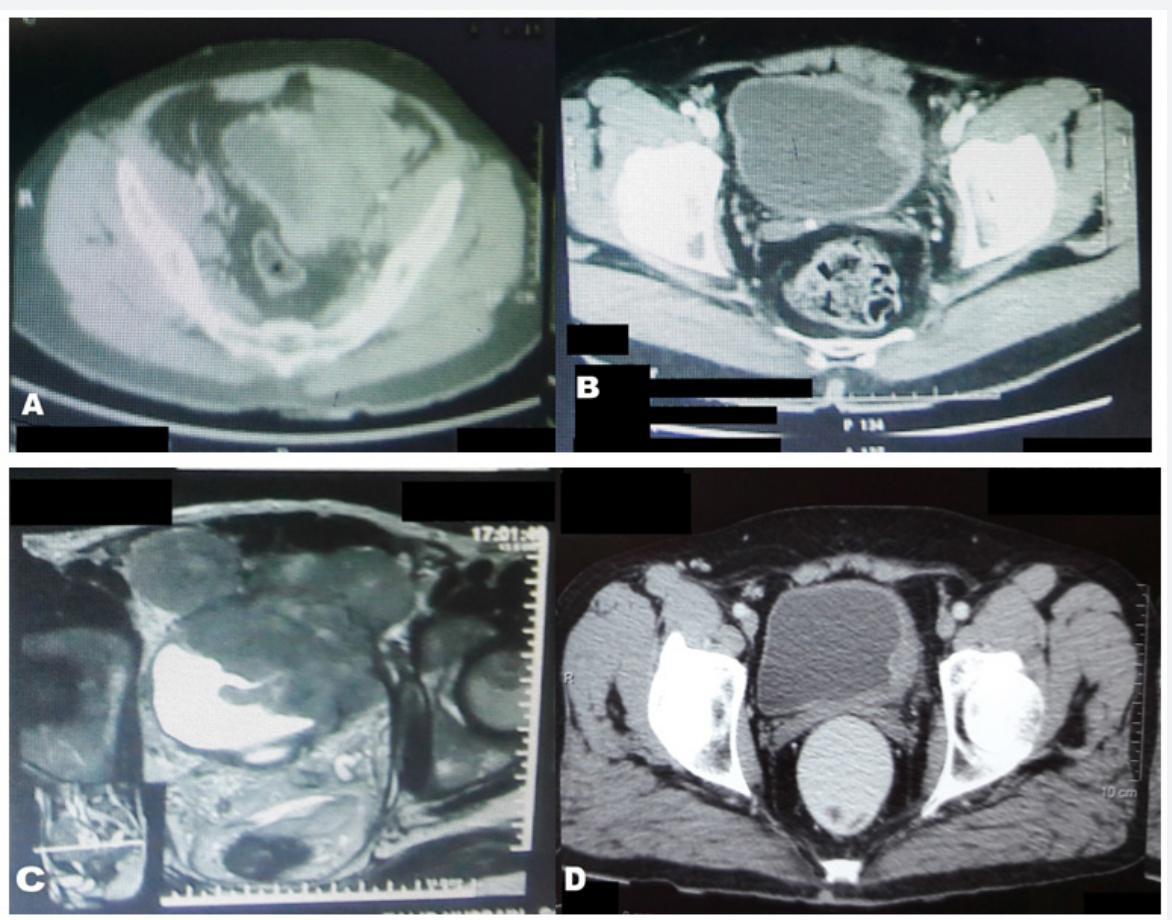

\section{Figure 1:}

A. CT scan pelvis showing bladder growth involving anterior and left lateral walls extending up to pelvic side wall.

B. CT scan pelvis showing bladder growth involving left lateral wall.

C. CT scan pelvis showing iliac nodal mass.

D. CT scan pelvis showing smooth thickening of anterolateral wall of urinary bladder.

To resolve the discrepancy between the initial and repeat histopathology reports, histopathology slide review with immunohistochemistry was advised. The diagnosis of small cell neuroendocrine carcinoma was made. Immunohistochemistry showed positive staining for CD56 and Synaptophysin while CKAE1/AE3 was weakly positive and Cam 5.2 was negative. CT scan chest showed bronchiectatic and emphysematous changes associated with consolidation. Patient was discussed in tumor board and was planned for combination chemotherapy cisplatin and etoposide. Initially patient responded well to chemotherapy and interim assessment done showed improvement in the disease process but after completion of six cycles CT scan showed progression of the disease. The patient's performance status worsened (ECOG 3) and he developed hematuria which was initially settled. Patient was discussed in intradepartmental meeting and was planned for palliative radiotherapy with total dose of 30 Gy in 10 fractions. As the patient had poor compliance he didn't turn up for radiotherapy on scheduled date and was lost to follow up.

\section{Case 2}

A 32 year old male with no known comorbids and with family history of skin cancer presented with complaint of painless hematuria for one and a half year. Initially patient was investigated and treated at urology department. His TURBT was done and histopathology revealed high grade papillary carcinoma with muscle invasion. He remained well for short time but again developed hematuria, burning micturition and backache. Second TURBT was done and histopathology report favored low grade muscle invasive carcinoma with intermediate lymphovascular invasion.

Patient was then referred to Oncology department. Upon examination patient's ECOG performance score was 2. He was pale and had generalized body weakness with tenderness in pelvic region. Abdominal examination was unremarkable. Histopathology slide review was done. Final diagnosis of small cell neuroendocrine carcinoma was made. Immunohistochemistry showed positivity for Synaptophysin and GATA-3. Further CT 
scan was done that showed an irregular shaped heterogeneously enhancing soft tissue mass lesion with internal low density areas arising from left lateral wall of urinary bladder (Figure 1B). The size of the mass was $7.6 \times 4.4 \times 7.2 \mathrm{~cm}$. Fat planes were indistinct with rectus muscles and there was presence of the iliac nodal mass of $3 \times 2.5 \mathrm{~cm}$ (Figure 1C). Pleural based lung nodules were present suggestive of lung metastasis.

Bone scan for skeletal assessment was negative for bone metastasis but showed inflammatory and arthritic changes. The case was discussed in tumor board and patient was planned for combination chemotherapy cisplatin and etoposide. Patient responded well to 1st cycle of chemotherapy but not to the second and third cycle. U/S abdomen and pelvis showed progression of the disease. Patient was radiated in the palliative setting with total dose of 30 Gy in 10 fractions. Octreotide scan was done which showed no scan evidence of Tc-99 avid lesion. Patient was explained thoroughly about poor prognosis and was kept on best supportive treatment.

\section{Case 3}

52 years old male, hypertensive, chronic smoker and addicted to niswar for 40 years presented with the complaint of painless hematuria and flank pain for 6 months. The patient was initially investigated and treated at urology department. His baseline ultrasound was done that showed bladder growth. TURBT was done and histopathology showed papillary urothelial carcinoma with muscle invasion. Histopathology blocks were reviewed and immunohistochemistry was done. Microscopy showed diffuse sheets of monotonous looking cells. Individual cells were polygonal with stippled chromatin pattern. Tumor cells were positive for CK7, CD56, Synaptophysin, Chromogranin, NSE, and focal weak positive for Cam 5.2. The cells were negative for CK20 and PSAP. Ki67 index was 90\%.Pathological diagnosis of small cell neuroendocrine tumor with muscle and lymphovascular invasion was made. Patient was referred to Oncology department.

Post-operative CT scan was advised that showed partially distended urinary bladder with mild focal smooth thickening of anterolateral wall measuring up to $6.2 \mathrm{~mm}$ (Figure 1D). Metastatic work up was negative for any distant metastasis. Octreotide scan showed no avid lesion. Patient was planned for combination chemotherapy with cisplatin and etoposide. After 2 cycles patient had mild subjective improvement.

\section{Discussion}

Urinary bladder is the most common site after lungs for small cell neuroendocrine tumors [2]. Small cell neuroendocrine carcinoma of urinary bladder was described for the first time by Cramer et al. [4]. The neuroendocrine tumors behave more aggressively and have poorer prognostic outcomes when compared with other histological subtypes [2]. Moreover pure forms of small cell tumors have poor prognosis as compared to mixed forms [5]. Mean age at the time of diagnosis is 66 years and male to female ratio is 5:1 [2]. In contrary our patients were 32,41 and 52 year old.

Cigarette smoking is considered as an established risk factor in 50-70\% patients of SCNC [3]. Bladder calculi and chronic cystitis also contribute to the increased risk [2]. Our patients had associated risk factors including family history of malignancy, bladder stones, and addictions like smoking, betel nut and niswar. Gross morphology and presenting symptoms are similar to transitional cell carcinoma but it is more aggressive and has poor survival [6]. SCLC is usually muscle invasive [3] and more than $60 \%$ of the patients develop distant metastasis during the disease course [7]. Most frequent sites of metastasis are liver, lung, bone, brain and lymph nodes [2]. 67-100\% patients usually present with complaints of macroscopic hematuria. Other possible symptoms include dysuria, ureteral obstruction, abdominal pain, weight loss and recurrent UTIs [3]. Common sites of involvement are lateral side walls and dome of bladder6. Most patients have distant metastasis (24\%) and lymph node metastasis (5\%) at initial presentation6. Prognostic outcome is $19 \%$ when combined for all four stages [8]. Our patients presented with dysuria and hematuria. Two of the patients had nodal involvement and one had pulmonary metastasis.

The histology of the tumor cells is similar to small cell lung cancer due the presence of sheets of atypical small cells with scent cytoplasm and characteristic "salt and pepper" chromatin, high mitotic ratio and crush artifacts and vascular invasion [2]. Panel of immunohistochemical stains usually employed for diagnosis are Synaptophysin, CD56 and Chromogranin A and CK7 [2,9]. NSE although nonspecific is positive in about $80 \%$ of the cases while TTF- 1 and Cam 5.2 can also be positive [2,9]. These tumors always have high Ki-67 proliferation index of more than $50 \%$. Patients with Ki-67 index of up to $55 \%$ have poor response to chemotherapy but better survival as compared to patients with Ki-67 index of more than 55\%. This shows survival is unaffected by platinum based chemotherapy in advanced neuroendocrine tumors [10]. Our patients showed positivity for Synaptophysin, Chromogranin, CK7, CD56, GATA-3, NSE, CKAE1/AE3 and weak positivity for Cam5.2. Ki-67 index done in one patient was $90 \%$. Due to the morphological resemblance with transitional cell carcinoma, in all the three patients, SCNC was diagnosed after review of histopathology and immunohistochemistry, initially diagnosis was missed.

Definitive recommendations for the management of small cell neuroendocrine bladder tumors have yet not been established. Management plan can vary according to patient's performance status and stage of the disease [3]. Combination of surgery, chemotherapy and radiotherapy is usually employed while treating this rare cancer [3]. TURBT alone is not curative but in combination with chemotherapy and radiation is considered superior over radical cystectomy [3]. Surgery is usually reserved for early stage cancers [3]. Our patients underwent TURBT, chemotherapy and palliative radiotherapy. 
Chemotherapy combination of etoposide and platinum based drugs is the treatment of choice both in adjuvant and neoadjuvant setting [9]. Role of neoadjuvant chemotherapy is well established in surgically operable cases and has a 5 year survival of $78 \%$ as compared to $36 \%$ in patients in which surgery alone has been done [11]. There is a questionable role of chemotherapy in adjuvant setting but is still recommended in stage III and IV patients [9]. Patients with progressive and relapsed disease might get some benefit from second line chemotherapy regimens and newly emerging targeted therapies [9]. In our chemotherapy given cases patient's performance status deteriorated even with first line chemotherapy so they were not considered fit for any further chemotherapy. The best possible option was salvage radiotherapy.

Radiation with radical dose and with concurrent chemotherapy can be given in patients with early stage disease [9]. Concurrent chemo radiation is curative and has more promising results as compared to radiation alone with 5 year survival of $28-70 \%[8,9]$. Radiotherapy is usually indicated in inoperable cases and patients who wish to preserve the bladder [8]. As patients usually present is advanced stages and with poor performance status in SCLC, 5 year survival in stage III and IV disease is $15.4 \%$ and $10.5 \%$ respectively [9]. Palliative radiotherapy is also indicated in patients who present with brain and bone metastasis or spinal cord compression [9]. Our patients presented in advanced stage and after initial poor response to chemotherapy, aim of our treatment was palliative with hypo fractionated dosage of 30 Gy in 10 fractions in 2 weeks' time for localized disease control.

\section{Conclusion and Recommendations}

Small cell neuroendocrine carcinomas are rare primary bladder tumors which usually present in advanced stages and have poor therapeutic response. Histopathologists should report carefully keeping in mind rare malignancies. Patients not responding to combination chemotherapy can be considered for hypo fractionated palliative radiotherapy. More research is required to look for optimal management of these patients.

\section{Conflicts of Interest}

There are no conflicts of interest.

\section{References}

1. Kouba E, Cheng L (2016) Neuroendocrine Tumors of the Urinary Bladder According to the 2016 World Health Organization Classification: Molecular and Clinical Characteristics. Endocr Pathol 27(3): 188-99.

2. Ulamec M, Krušlin B (2016) Neuroendocrine tumors in the urinary bladder. Endocrine oncology and metabolism 14: 42-49.

3. Sehgal SS, Wein AJ, Bing Z, Malkowicz SB, Guzzo TJ (2010) Neuroendocrine Tumor of the Bladder. Reviews in Urology 12(4): e197-e201.

4. Cramer SF, Aikawa M, Cebelin M (1981) Neurosecretory granules in small cell invasive carcinoma of the urinary bladder. Cancer 47(4): 724-730.

5. Ismaili N, Heudel PE, Elkarak F, Kaikani W, Bajard A, et al. (2009) Outcome of recurrent and metastatic small cell carcinoma of the bladder. BMC Urol 9: 4.

6. Fotopoulos G, Pentheroudakis G, Ioachim E, Pavlidis N. A patient with neuroendocrine carcinoma of the urinary bladder and paraneoplastic degenerative parencephalitis: A case report and review of the literature. Cancer Treatment Communications 2(1): 8-11.

7. Çamtosun A, Çelik H, Altıntaş R, Akpolat N (2015) Primary Small Cell Carcinoma in Urinary Bladder: A Rare Case. Case reports in urology 789806: 3.

8. Bansal A (2015) Changing trends in the management of small cell carcinoma of urinary bladder. Clinical Cancer Investigation Journal 4(4): 487-491.

9. Ismaili N (2011) A rare bladder cancer-small cell carcinoma: review and update. Orphanet journal of rare diseases. 6(1): 75.

10. Sorbye H, Welin SF, Langer SW, Vestermark LW, Holt N, et al. (2012) Predictive and prognostic factors for treatment and survival in 305 patients with advanced gastrointestinal neuroendocrine carcinoma (WHO G3): the NORDIC NEC study. Ann Oncol 24(1): 152-160.

11. Siefker-Radtke AO, Dinney CP, Abrahams NA, Moran C, Shen YU, et al (2004) Evidence supporting preoperative chemotherapy for small cell carcinoma of the bladder: a retrospective review of the MD Anderson cancer experience. The Journal of urology 172(2): 481-484.

Your next submission with Juniper Publishers will reach you the below assets

- Quality Editorial service

- Swift Peer Review

- Reprints availability

- E-prints Service

- Manuscript Podcast for convenient understanding

- Global attainment for your research

- Manuscript accessibility in different formats ( Pdf, E-pub, Full Text, Audio)

- Unceasing customer service

Track the below URL for one-step submission https://juniperpublishers.com/online-submission.php 